\title{
Thermal-wave investigation of physical and geometrical non-uniformities within thin layer chromatography (TLC) stationary phases
}

\author{
by Z. Suszyński*, R. Świta* and Joanna Łoś** \\ * Koszalin Univ. of Technology, 75-453, Śniadeckich 2, Koszalin, Poland, zbigniew.suszynski@tu.koszalin.pl \\ ** State Higher Vocational School in Koszalin, Leśna 1, 75-582 Koszalin, Poland
}

\begin{abstract}
The main goal of this paper is to demonstrate that photothermal method is an effective tool for detection of geometrical and structural non-uniformities within optically non-opaque, light scattering, planar multilayer structures. The investigated objects were plates of glass coated with silica, cellulose, aluminum oxide, polyamide or octadecylsilane. The thickness of coating ranged from 100 to $250 \mu \mathrm{m}$. IR active thermography measurements allow detecting and recognizing typical non-uniformities such as irregularity in thickness, absorption coefficients and physical structure of stationary phase.
\end{abstract}

\section{Introduction}

The quality of the chromatographic plates used in the TLC technology significantly affects the results of the chemical analysis. Layer thickness of the stationary phase, optical inhomogeneity and delamination affect the retention of the mobile phase and deform the spots determining the quantitative content of the sample. Visual assessment of plates is not sufficient to assess their quality. In the case of opaque structures also optical methods based on measurement of light reflected or passing-thru fail. In this article we propose the active thermography method for detection and identification of physical and geometrical non-uniformities in TLC plates. The method is fast and very accurate. It enables measurements of non-opaque, light scattering, planar multilayer structures. Photo-thermal methods are widely used for detecting, identification and quantifying optical, thermal, geometrical and structural features of solids. There are few methodologies that are based on photo-thermal phenomenon: photoacoustics, thermal-wave microscopy, active thermography and thermovision. The common principle of those methods lies in energetic stimulation of the examined objects and observing their temperature field in time or frequency domain. Dynamic temperature field depends on spatiotemporal distribution of dissipated power and thermal impedance of the medium:

$$
T(x, y, z, t)=F\left\{P(x, y, z, t), Z_{t h}(x, y, z, t)\right\}
$$

This implies a complex relationship between temperature (or temperature contrasts) and physical properties of investigated object. In some cases the detection and measurement of selected properties may be impossible due to the low sensitivity of the temperature field of the selected physical parameters. Despite this, the unambiguous interpretation of measurement results for many types of objects is possible [1,2].

When temperature detection uses the IR sensors the properties of the object in the infrared diapason should additionally be taken into account [3]. This may complicate in a significant way the quantitative analysis of the physical properties of the tested objects.

The main areas of application of the photo-thermal methods are investigating the spectral and thermal properties of solids, detection of selected physical traits and density of dissipated power distribution.

\section{Description of the problem and methodology}

The stationary phases for the separation of analytes are porous, optically non-opaque structures having a thickness from 100 to 250 micrometers. These materials are complex both in terms of structure and chemical composition and their optical and thermal properties can vary from sample to sample. Stationary phases belong to materials of poor thermal conductivity and their density is of order of a few hundreds of $\mathrm{kg} \cdot \mathrm{m}^{-3}$. They are made among others of silicate and cellulose. Very often, stationary phase is deposited on glass.

Changes in structure, thickness and optical heterogeneity affect the separation of analytes and may make their detection difficult. That is why the structural, optical and thickness homogeneity is a desirable feature of stationary phases.

Active thermovision with optical excitation seems to be an appropriate tool for detecting mentioned above traits - optical properties affect density and spatial distribution of power excitation while thickness and physical and chemical structure have impact on resultant thermal impedance.

Therefore, the purpose of our research was to examine and identify the inhomogeneity in selected stationary phases that are available on the market. 


\section{Experiment setup}

The stationary phases for the separation of analytes are porous, optically non-opaque structures of thickness ranging from 100 to 250 micrometers. These materials are complex both in terms of chemical composition and their optical and thermal properties. Changes in structure and thickness of active layer affect the separation of analytes. For this reason these parameters should be monitored.

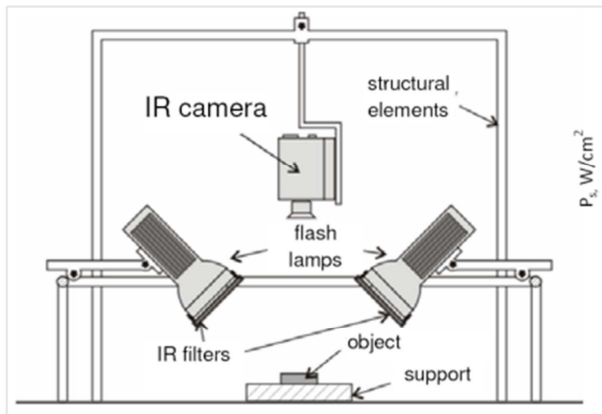

a)

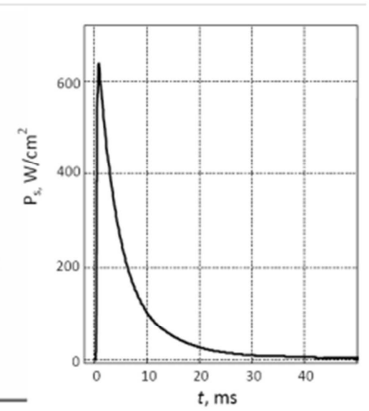

b)

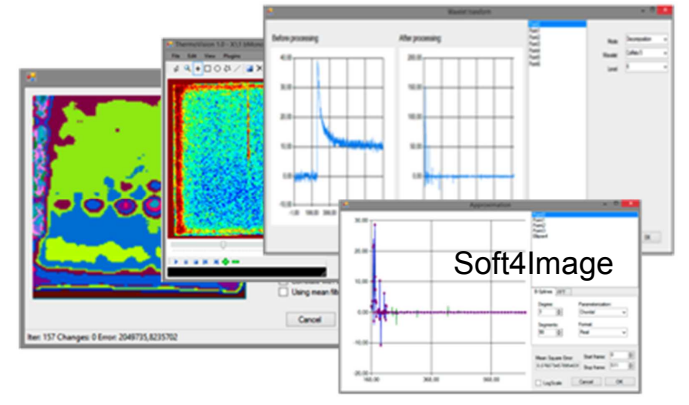

c)

Fig. 1. Experimental set-up (a) and power density pulse of excitation (b), software (c).

Data acquisition and signal processing protocols are based on active pulse thermo-vision system presented in fig.1. The source of energy is composed of six flash lamps providing the total power density of $600 \mathrm{~W} \cdot \mathrm{cm}^{-2} \mathrm{for} 10 \mathrm{~ms}$ pulse in visible spectra diapason. Temperature field is registered by fast IR camera with resolution of $128 \times 128$ pixels captured with $500 \mathrm{fps}$. Temperature sequence has been processed with computer program Soft4image ver.1.0.

\section{Model and results of measurements}

The cross-section of investigated TLC plates is shown in fig. 2.

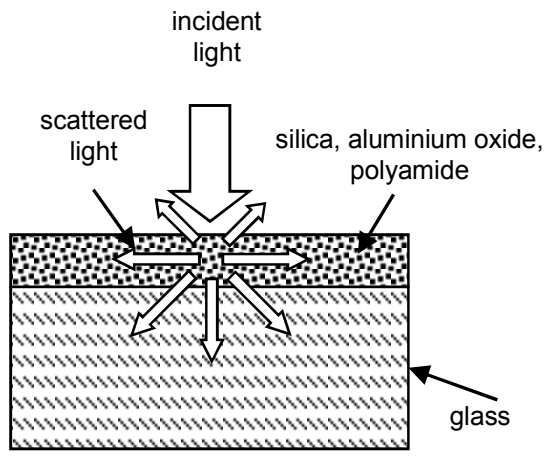

a)

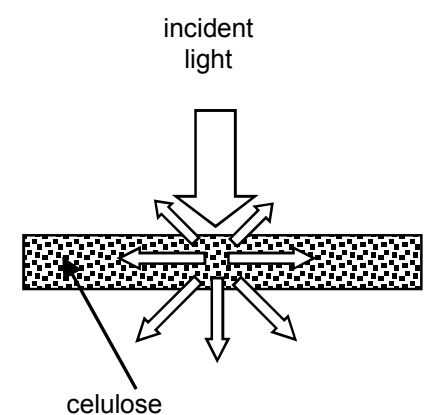

b)

Fig. 2. Cross-section of thin layer chromatography plates

There are single and double-layered structures. The first layer thickness ranges from 100 to $250 \mu \mathrm{m}$. It scatters visible and IR radiation as well as is characterized by low thermal conductivity and low density. The second layer is usually made of glass that is transparent for visible and opaque for IR radiation.

Similar structures were described in the papers devoted to the possibility of simultaneous measurement of thermal diffusivity and optical absorption coefficient using photo-thermal methodology [4]. Unfortunately, the models presented in those papers do not relate directly to the light-scattering materials. However, the changes in the intensity of the radiation versus depth for both processes have the same form and are identical when $\beta=N \sigma_{s}$ (equations (1) and $(2))$ : 


$$
\begin{gathered}
I_{a}(z)=I(z=0) \cdot \exp (-\beta z) \\
I_{s}(z)=I(z=0) \cdot \exp \left(-N \sigma_{s} z\right)
\end{gathered}
$$

where:

$\beta$ - optical absorption coefficient,

$\mathrm{N}$ - density of scattering centers,

$\sigma_{\mathrm{s}^{-}}$cross section area of centers.

That is why for qualitative analysis of measurements model developed for homogeneous optically non-opaque materials has been used (fig.2)

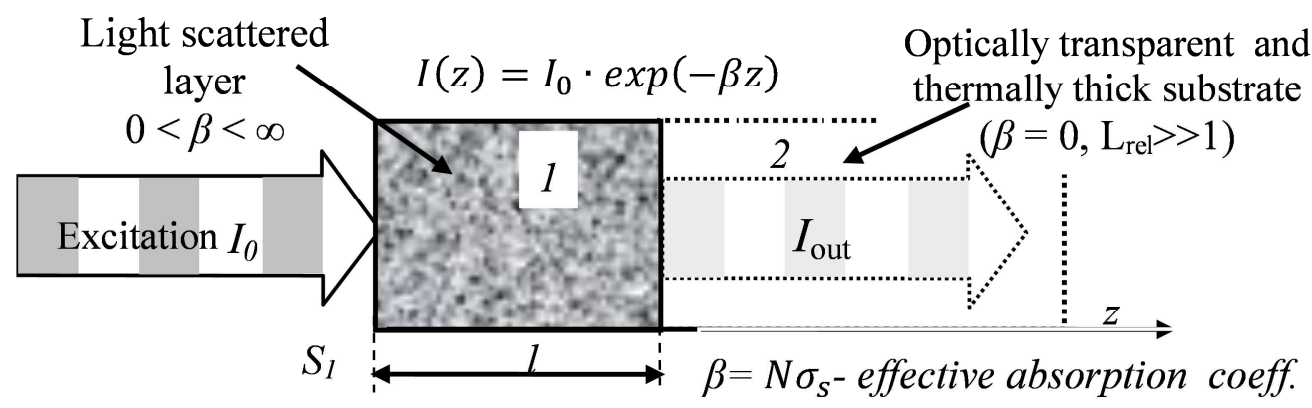

Fig.2. Simplified physical model of analysed structures

For such a structure complex temperature at the surfce $S_{1}$ for harmonic excitation is given by the formula:

$$
\Theta_{1}=I \cdot Z_{c}\left[\frac{\left(\operatorname{ch} \Gamma-\exp \left(-\beta_{L}\right)\right)\left(1-(1+j) \frac{L_{r e l}}{\beta_{L}} \varepsilon_{r e l}\right)-\operatorname{sh} \Gamma\left((1+j) \frac{L_{r e l}}{\beta_{L}}-\varepsilon_{r e l}\right)}{\left(1-2 j \frac{L_{r e l}^{2}}{\beta_{L}^{2}}\right)\left(s h \Gamma+\varepsilon_{r e l} \operatorname{ch} \Gamma\right)}\right]
$$

where:

$I$ - power density of incident light,

$\beta_{L}=\beta \cdot l, L_{r e l}=l \cdot \mu^{-1}$,

$\Gamma=(1+i) \cdot L_{r e l}$

$\varepsilon_{\text {rel }}=\varepsilon_{1} \cdot \varepsilon_{2}^{-1}$

$\mu=\sqrt{2 \cdot \alpha \cdot \omega^{-1}}$

$\alpha$ - thermal diffusivity of the first layer,

$\varepsilon$ - thermal effusivity.

Main parameters that have impact on temperature $\Theta_{1}$ are relative thickness $L_{\text {rel, }}$ normalized absorption coefficient $\beta_{\mathrm{L}}$ of first layer and relative effusivity $\varepsilon_{\text {rel }}$. These parameters are related to basic thermal parameters (thermal conductivity, capacity and density) of both layers and normalized optical absorption coefficient and thickness of the first layer. 


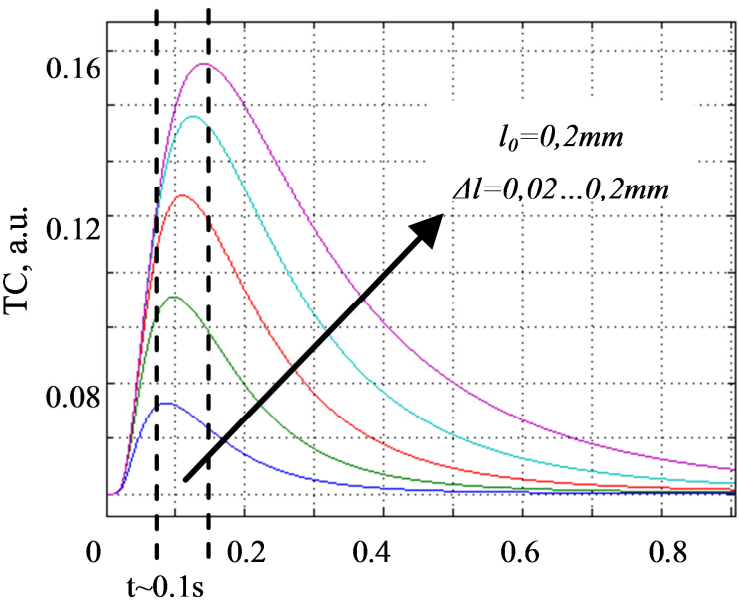

a)

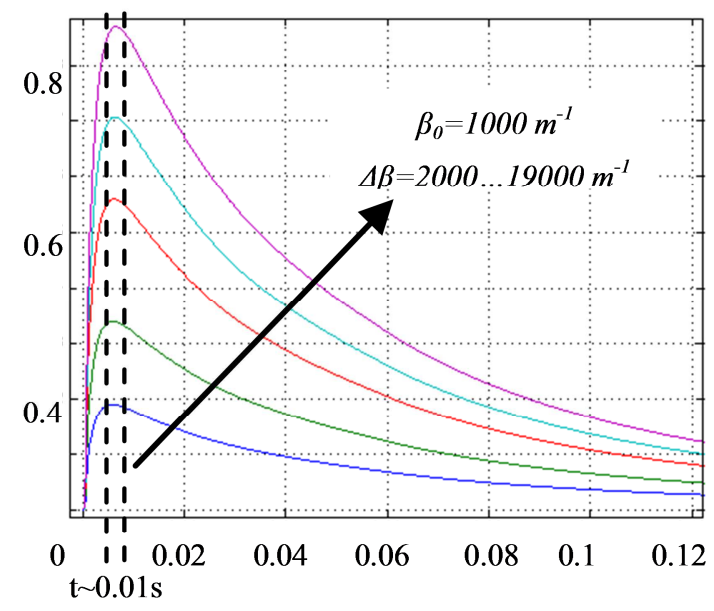

b)

Fig.3. Calculated temperature contrasts caused by increase in thickness (a) and absorption coefficient (b)

Using formula (4) and Fourier transform, the temperature response and temperature contrasts signal versus time for areas of increased thickness and increased absorption for pulse excitation (form presented in fig.1b) have been calculated (fig. $3 \mathrm{a}, \mathrm{b})$

These diagrams show that the contrast caused by the increase in the thickness of the stationary phase lasts from 0.2 to $0.4 \mathrm{~s}$ and its maximum occurs approximately $0.1 \mathrm{~s}$ after excitation. Contrast caused by rising absorption is shorter (it lasts $\sim 40 \mathrm{~ms}$ ) and its maximum value appears for approx. 10ms from the beginning of stimulation. The difference between both kind of contrasts can be easily explained, because in the first case thermal capacity of stationary phase increases (layer 1), while highest value of absorption causes first of all increase of dissipated energy and magnitude of temperature.

In fig. 4a we can see experimental contrasts of selected areas (1, 2, 3). Measurement was performed for stationary phase (plate of glass coated with silica gel of the thickness of $0.2 \mathrm{~mm}$ ) with spots of fullerenes C60/C70 of different mass ranged from 10 to $200 \mathrm{ng}$. Its presence caused slight increase of absorption and did not affect thermal properties. The external right and bottom areas are characterized by increased thickness being result of meniscus. Contrast pulses of areas no. 2 and 3 are shorter then contrast of the area no.1. The basic properties of both contrasts are similar to contrasts determined from model. Basing on it and on the knowledge about properties of stationary phase one can conclude, that also area no. 3 is characterized by increased optical absorption.

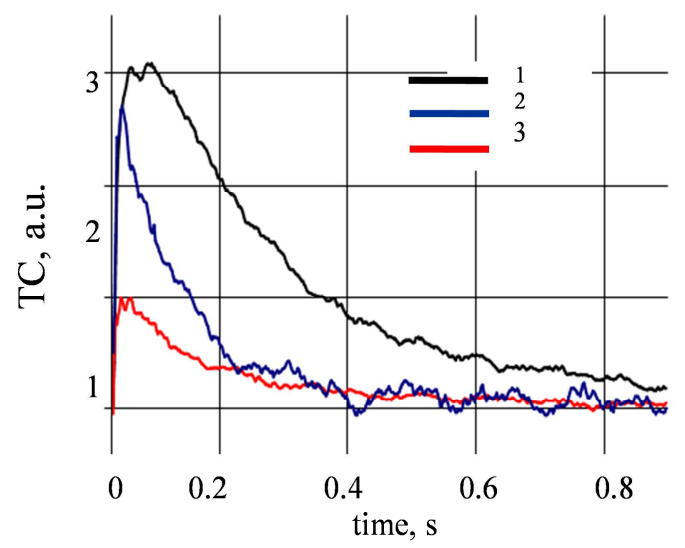

a)

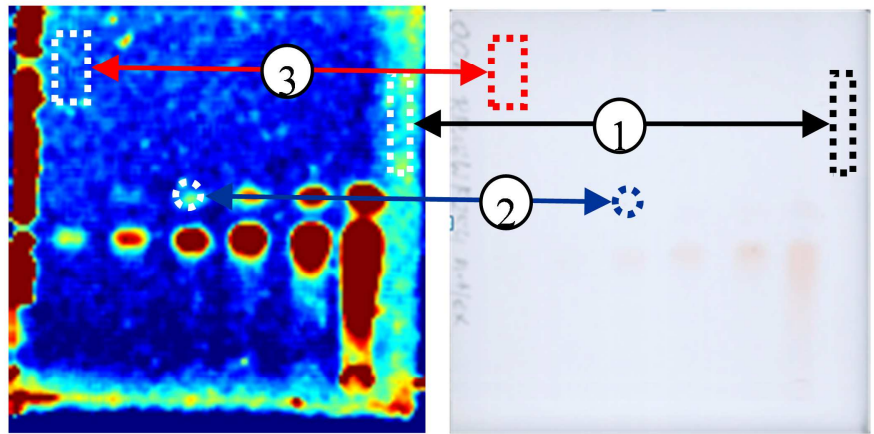

b)

c)

Fig. 4. Temperature contrasts of non-uniformities in time domain (a), temperature image (b) and optical image of TLC plate (c) 
10.21611/qirt.2016.129

\section{Conclusions}

Thermal-wave methods can be effective tool for detection and qualification of optical and geometrical inhomogeneities in non-opaque, light scattering, planar multilayer structures like a stationary phases used for TLC. Active thermovision method allows for very fast evaluating of optical and geometrical uniformity and makes it possible to recognize the reasons of temperature contrasts. The growing absorption coefficient causes increase of temperature magnitude while increase of the stationary phase thickness lengthens duration of contrast pulse.

\section{REFERENCES}

[1] 1. H. Qu, C. Wang i X. M. A. Guo, Reconstruction of depth profiles of thermal conductivity of case hardened steels using a three-dimensional phototermal technique, J. Appl.Phys, 104, pp. 113518/1-9, 2008.

[2] 2. Z. Suszyński, P. Majchrzak, Photo-thermal depth profiling of metal-ceramics structures using artificial neural networks, J.de Physique IV France 117, pp. 91-94.

[3] 3. J. Ravi, S. Longuemart, S. Paoloni and others, Optothermal depth profiling by neutral network infrared radiometry signal recognition, J. of Appl. Phys., vol. 97, 2005, pp.014701-1-7.

[4] 4. Z. Suszyński, M. Maliński, L. Bychto, Thermal Parameters Measurement Method of Electronics Materials, IEEE Trans. On Component Packaging and Manufacturing technology - Part A, 21(3),1998,pp. 424-433. 